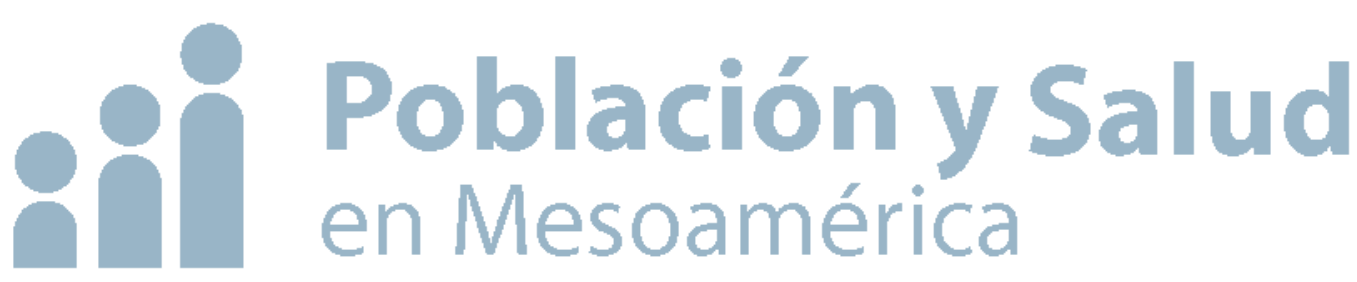

\title{
Tendência e diferenças regionais de homicídios masculinos por arma de fogo em um estado da Amazônia Legal, Brasil
}

Franciele Silvia de Carlo, Ligia Regina de Oliveira y Amanda Cristina de Souza

\section{Como citar este artículo:}

De Carlo, F., de Oliveira, L. y de Souza, A. (2021). Tendência e diferenças regionais de homicídios masculinos por arma de fogo em um estado da Amazônia Legal, Brasil Población y Salud en Mesoamérica, 18(2). Doi: https://doi.org/10.15517/psm.v18i2.43577

\section{(1)@(๑)}

ISSN-1659-0201 http://ccp.ucr.ac.cr/revista/

Revista electrónica semestral

Centro Centroamericano de Población Universidad de Costa Rica 


\title{
Tendência e diferenças regionais de homicídios masculinos por arma de
}

$$
\text { fogo em um estado da Amazônia Legal, Brasil }
$$

Regional trends and differences in male homicides by firearms in a state in the Legal Amazon, Brazil

Tendencias y diferencias regionales en los homicidios masculinos por armas de fuego en un estado de

\author{
la Amazonia Legal, Brasil
}

\section{Franciele Silvia de Carlo' Ligia Regina de Oliveira² Amanda Cristina de Souza Andrade ${ }^{3}$}

\begin{abstract}
Resumo:
Objetivos: Analisar a tendência e projetar a taxa de homicídios por arma de fogo em homens jovens, segundo macrorregião de saúde, no estado de Mato Grosso, Brasil. Metodologia: Estudo ecológico, exploratório, de tendência temporal que utilizou dados do Sistema de Informação sobre Mortalidade. Taxas de mortalidade padronizada pela população mundial foram calculadas. Para modelagem da série e predição da taxa de homicídio por arma de fogo para o período de 2018 a 2022 utilizou-se o modelo auto regressivo de ordem um - AR (1). Resultados: A macrorregião Centro Norte apresentou as maiores taxas de mortalidade por arma de fogo na maior parte dos anos. A menor taxa do período ocorreu em 2013, na macrorregião Leste. Assim como todo o estado de Mato Grosso, as macrorregiões Norte, Oeste, Sul apresentaram tendência crescente e significativa, enquanto nas macrorregiões Leste, Centro Norte e Centro Noroeste as taxas se mantiveram estáveis. Conclusão: A mortalidade de jovens do sexo masculino por armas de fogo foi elevada especialmente nas macrorregiões mais urbanizadas, com conflitos agrários e região de fronteira. Se as estratégias não forem mudadas, as taxas de homicídios de homens jovens por armas de fogo no Mato Grosso continuarão altas e/ou crescentes.
\end{abstract}

Palavras-chave: Homicídio; Violência com arma de fogo; Disparidades nos níveis de saúde; Homens.

Abstract: Objectives: To analyze the trend and project the firearm homicide rate in young men, according to the health macro-region, in the state of Mato Grosso, Brazil. Methodology: Ecological, exploratory, time trend study that used data from the Mortality Information System. Mortality rates standardized by the world population were calculated. To model the series and predict the firearm homicide rate for the period from 2018 to 2022, the order one autoregressive model - AR (1) was used. Results: The Centro Norte macro-region had the highest firearm mortality rates in most years. The lowest rate in the period occurred in 2013, in the East macro-region. As with the entire state of Mato Grosso, the North, West, South macroregions showed an increasing and significant trend, while in the East, Central North and Central Northwest macroregions the rates remained stable. Conclusion: The mortality of young males by firearms was high especially in the most urbanized macro-regions, with agrarian conflicts and the border region. If the strategies are not changed, homicide rates of young men by firearms in Mato Grosso will continue to be high and/or increasing.

Keywords: Homicide, Gun violence, Health Status Disparities, Men.

Resumen: Objetivos: Analizar la tendencia y proyectar la tasa de homicidios por arma de fuego en hombres jóvenes, según la macrorregión de salud, en el estado de Mato Grosso, Brasil. Metodología: Estudio ecológico, exploratorio, de tendencia temporal que utilizó datos del Sistema de Información de Mortalidad. Se calcularon las tasas de mortalidad estandarizadas por la población mundial. Para modelar la serie y predecir la tasa de homicidios por arma de fuego para el período de 2018 a 2022, se utilizó el modelo autoregresivo de orden uno: AR (1). Resultados: La macrorregión Centro Norte tuvo las tasas de mortalidad por armas de fuego más altas en la mayoría de los años. La tasa más baja del período se produjo en 2013, en la macrorregión Este. Al igual que en todo el estado de Mato Grosso, las macrorregiones Norte, Oeste y Sur mostraron una tendencia creciente y significativa, mientras 
que en las macrorregiones Este, Centro Norte y Centro Noroeste las tasas se mantuvieron estables. Conclusión: La mortalidad de varones jóvenes por armas de fuego fue alta especialmente en las macrorregiones más urbanizadas, con conflictos agrarios y la región fronteriza. Si no se cambian las estrategias, las tasas de homicidio de hombres jóvenes por armas de fuego en Mato Grosso seguirán siendo altas y/o en aumento.

Palabras-clave: Homicidio, Violencia con armas, Disparidades en el estado de salud, Hombres.

Recibido: 27 ago 2020 | Corregido: 01 dic 2020| Aceptado: 4 dic 2020

'Instituto de Saúde Coletiva, Universidade Federal do Mato Grosso - UFMT. Cuiabá, Mato Grosso, Brasil. fsC mt@yahoo.com.br ORCID:

http://orcid.org/0000-0002-3217-5273.

2Instituto de Saúde Coletiva, Universidade Federal do Mato Grosso - UFMT. Cuiabá, Mato Grosso, Brasil. Irdoliveira@gmail.com. ORCID:

http://orcid.org/0000-0002-7325-1391.

${ }^{3}$ Instituto de Saúde Coletiva, Universidade Federal do Mato Grosso - UFMT. Cuiabá, Mato Grosso, Brasil.

amandasouza_est@yahoo.com.br_ORCID: http://orcid.org/0000-0002-3366-4423. 


\section{Introdução}

O Relatório de Estatísticas Globais de Saúde (World Health Statistics), publicado em 2017 pela OMS, estima que, a cada ano, mais de 1,4 milhões de pessoas morrem devido à violência no mundo, sendo a principal causa o homicídio, que registra anualmente cerca de 464.000 óbitos (United Nations Office On Drugs And Crime [UNODC], 2019).

Homens e adolescentes do sexo masculino com idade entre 15 e 29 anos estão em maior risco de homicídio globalmente. Isso se deve, em grande parte, à situação nas Américas, onde os causadores da violência homicida são frequentemente o crime organizado e os ferimentos por armas de fogo são a causa mais frequente de morte - fatores que são conhecidos por favorecer a prevalência de homens jovens como vítimas e perpetradores (Ministério dos Direitos Humanos, 2018; UNODC, 2019).

Em 2017, o Brasil era o sétimo no ranking dos países com maior taxa de mortalidade por homicídios das Américas (31,6/100.000). Honduras $(55,7 / 100.000)$ e Venezuela $(49,2 / 100.000)$ eram os mais violentos, enquanto o Canadá tinha as menores taxas de assassinatos, com índice de 1,5 homicídios para cada 100 mil habitantes. Entre os países da América do Sul, o Brasil só é menos violento que Colômbia $(43,1 / 100.000)$, e Venezuela (49,2/100.000). Outros países de todo continente americano que apresentam as menores taxas incluem Chile (4,4/100.000), Cuba (5,5/100.00) e Argentina (6,2/100.00) (World Health Organizacion [WHO], 2018).

Quanto ao meio empregado para ocorrência desses homicídios, mais da metade de todos os homicídios no mundo foram cometidos com armas de fogo, enquanto apenas $20 \%$ envolveu objetos pontiagudos em 2017. Nas Américas, as armas de fogo estiveram envolvidas em cerca $75 \%$ dos homicídios naquele mesmo ano. Alguns países com altas proporções de mortes por armas de fogo tendem a ter altas taxas de homicídio e países onde objetos pontiagudos são o principal mecanismo de morte tendem a ter baixas taxas de homicídio, o que sugere que armas de fogo e as taxas de homicídio estão relacionadas (UNODC, 2019). 
No Brasil, foram registrados 65.602 homicídios em 2017, destes, 33.772 (51,5\%) eram homens de 15 a 29 anos, tendo as armas de fogo representado 80\% (27.008) destas mortes. Em Mato Grosso, das 1.102 homicídios registrados nesse mesmo ano, 432 (39,2\%) ocorreram entre homens jovens, e destes, 73,1\% (316) teve como meio utilizado a arma de fogo (Ministério da Saúde, 2019).

Além disso, o estado de Mato Grosso apresentou crescimento ao longo dos anos da taxa de homicídios por armas de fogo em homens jovens, quando o estado registrou taxa de 48,9/100.000 em 1996 e de 65,3/100.000 em 2017, com variações importantes, como no ano de 2014 (97,4/100.000) (Ministério da Saúde, 2019).

Diante desta perspectiva, o objetivo deste estudo foi analisar a tendência e projetar a taxa de homicídios por arma de fogo em homens jovens, segundo macrorregião de saúde, no estado de Mato Grosso, Brasil.

\section{Referencial teórico}

O debate em torno da violência como problema de saúde tornou-se robustecida após a publicação do Relatório Mundial sobre Violência e Saúde em 2002, que definiu violência como sendo o "uso de força física ou poder, em ameaça ou na prática, contra si próprio, outra pessoa ou contra um grupo ou comunidade que resulte ou possa resultar em sofrimento, morte, dano psicológico, desenvolvimento prejudicado ou privação" (Krug et al, 2002, p. 05) provocando, a partir daí, maior desvelo sobre o tema. Complementarmente, Minayo (2013) refere que devido ao forte impacto físico e psicológico para pessoas e coletividades, a violência constitui-se como grave problema para saúde e é considerada um fenômeno social que perturba as relações sociais.

A violência encontra em si várias particularidades e nesse contexto o homicídio constitui sua expressão máxima, sendo definido como a morte ilegal intencionalmente causada a uma pessoa por outra pessoa (Krug et al., 2002).

Mundialmente, inúmeros estudos associam as leis de armas de fogo e a ocorrência e tentativas de homicídios; suas conclusões confluem para assertiva de que força da legislação sobre armas de fogo em 
geral, as leis relacionadas ao fortalecimento das verificações de antecedentes e da permissão para compra em particular estão associadas à diminuição das taxas de homicídio por armas de fogo (Simonetti et al., 2015; Kalesan et al., 2016; Lee et al., 2017; Zeoli et al., 2019).

O debate sobre as armas de fogo e o seu impacto na violência vem recebendo crescente atenção da produção intelectual no país. Em torno da discussão sobre a revogação do estatuto do desarmamento e relaxamento das regras para porte ou posse de armas as opiniões se dividem entre os que defendem medidas mais rígidas para a posse e o porte de armas de fogo, e aqueles que afirmam o direito dos cidadãos de possuí-las como forma de garantir sua própria segurança e defender seu patrimônio (Cerqueira et al., 2020). Todavia, à sombra dessa discussão, está o crescimento das taxas de homicídios causados por armas de fogo no Brasil, ao passo de que em 1996 a taxa registrada era de 14,6/100.000 e em 2017 já havia alcançado 31,6/100.000 homicídios (Ministério da Saúde, 2019)

As taxas crescentes de homicídios e seus efeitos negativos se estende à diversas áreas. A Declaração de Geneva admite o impacto econômico da violência armada, afirmando que o custo da perda da produtividade devido à violência por arma de fogo em situações de não conflito é de cerca de 95 bilhões de dólares anuais (podendo subir para 163 bilhões de dólares) em todo o mundo e, pode ainda, fazer o crescimento anual de uma economia típica cair em aproximadamente dois por cento (Geneva Declaration on Armed Violence and Development, 2019).

O documento ainda acrescenta que a violência armada pode provocar deslocamentos humanos forçados, erosão do capital social, destruição da infraestrutura, enfraquecer instituições públicas, facilitar a corrupção e proporcionar clima de impunidade (Geneva Declaration on Armed Violence and Development, 2019).

\section{Metodología}

\subsection{Enfoque}

Estudo ecológico, exploratório, de tendência temporal, realizado a partir da análise da taxa de homicídios provocados por arma de fogo. 


\subsection{População de estudo}

A população de estudo foram casos de homicídios em indivíduos do sexo masculino com idade entre 15 a 29 anos residentes nas Macrorregiões de Saúde do estado de Mato Grosso, Brasil. A faixa etária selecionada foi fundamentada mediante a literatura que atribui a maior vitimização por homicídios a homens de 15 a 29 anos (UNOCD, 2019).

\subsection{Coleta de dados}

Os dados foram coletados no Sistema de Informação sobre Mortalidade (SIM) no site do Departamento de Informática do Sistema Único de Saúde (DATASUS) do Ministério da Saúde do Brasil, de acesso livre na internet.

Trata-se de um órgão Ministério da Saúde do Brasil que coleta, processa e dissemina informações sobre saúde. O DATASUS recebe as informações do Sistema de Mortalidade (SIM), sendo este último alimentado pelas Declarações de óbito, documento nacionalmente padronizado que contém informações sociodemográficas e sobre o óbito (MELLO JORGE et al., 2009; Ministério da Saúde, 2011).

Para extração das informações sobre os óbitos no DATASUS, foram selecionados os dados de "mortalidade - 1996 a 2017, pela CID-10" - sendo 2017 o último ano com dados de livre acesso disponíveis no referido site à época da coleta de dados e selecionados os óbitos por agressões por meio de disparo de arma de fogo (X93 a X95). Os dados populacionais para o período de 1996 a 2017 também foram coletados no sítio eletrônico do DATASUS do Ministério da Saúde do Brasil e agrupados nas seis Macrorregiões de Saúde do estado.

\subsection{Processamento e análise dos dados}

A partir dos dados extraídos foram calculadas para cada ano as taxas brutas de mortalidade, dividindo-se o número de óbitos pela população masculina respectiva e multiplicada pela base referencial de 100.000 habitantes. Em seguida, foram calculadas taxas de mortalidade padronizadas por idade, para cada ano, de acordo com as macrorregiões de saúde e faixa etária, a fim de eliminar os efeitos de quaisquer distorções na distribuição etária. Para isso, utilizou-se o 
método direto, utilizando a população padrão mundial proposta por Segi (1960), modificada por Doll et al. (1966).

Para modelagem da série e predição da taxa de homicídio por arma de fogo para o período de 2018 a 2022 utilizou-se o modelo auto regressivo de ordem um - AR (1) aplicado a séries estacionárias. O valor da inclinação do modelo indica a tendência, crescente ou decrescente e se significativamente diferente de zero $(p<0,05)$.

Para cada macrorregião e para o estado foram estimados os correlogramas das funções de autocorrelação. Para todos os modelos foi verificado que os resíduos eram não correlacionados, utilizando-se o teste de Box e Pierce, que determina se um grupo de autocorrelações de uma série temporal é diferente de zero. Em vez de testar a aleatoriedade em cada atraso distinto, testa-se a aleatoriedade geral com base em vários atrasos (Box et al., 2015).

Os resultados foram apresentados por meio de tabelas e gráficos. Todas as análises foram realizadas no software STATA versão 12.0. O estudo foi aprovado pelo Comitê Permanente de Ética em Pesquisa da Universidade Federal do Mato Grosso, com parecer sob n 3.213.411/2019.

\section{Resultados}

De 1996 a 2017, ocorreram 5.791 homicídios por arma de fogo em jovens do sexo masculino no estado de Mato Grosso, representando 65,9\% entre todos os homicídios neste grupo populacional. Apesar de algumas variações e percentuais muito próximos ao longo dos anos, o ano mais recente (2017) registrou a menor taxa de homicídios por arma de fogo $(56,8 \%)$ no estado. A macrorregião de saúde Centro Norte registrou maior percentual médio (76,7\%) de homicídios por armas de fogo no período enquanto a macrorregião Leste apresentou o menor $(45,1 \%)$ (Tabela 1). 


\section{Tabela 1}

Percentual de homicídios por arma de fogo, segundo macrorregião de saúde. Mato Grosso, 1996-2017.

\begin{tabular}{|c|c|c|c|c|c|c|c|}
\hline \multirow[b]{2}{*}{ Ano do óbito } & \multicolumn{6}{|c|}{ Macrorregiões } & \multirow{2}{*}{$\begin{array}{l}\text { Mato } \\
\text { Grosso } \\
\text { (Total) }\end{array}$} \\
\hline & $\begin{array}{l}\text { Centro } \\
\text { Noroeste }\end{array}$ & $\begin{array}{l}\text { Centro } \\
\text { Norte }\end{array}$ & Leste & Norte & Oeste & Sul & \\
\hline 1996 & 68,8 & 74,5 & 83,3 & 51,9 & 90,6 & 68,8 & 73,3 \\
\hline 1997 & 55,6 & 54,0 & 64,3 & 66,7 & 78,0 & 57,7 & 60,1 \\
\hline 1998 & 81,0 & 69,1 & 44,4 & 82,1 & 74,4 & 63,2 & 70,6 \\
\hline 1999 & 75,0 & 70,7 & 50,0 & 51,6 & 69,2 & 55,6 & 66,4 \\
\hline 2000 & 50,0 & 83,2 & 51,6 & 55,6 & 66,7 & 67,6 & 72,5 \\
\hline 2001 & 54,5 & 74,1 & 76,5 & 65,4 & 43,5 & 64,7 & 69,3 \\
\hline 2002 & 56,0 & 77,1 & 68,0 & 57,4 & 58,6 & 71,1 & 69,2 \\
\hline 2003 & 68,3 & 73,7 & 35,0 & 56,4 & 69,6 & 55,9 & 66,5 \\
\hline 2004 & 67,5 & 77,3 & 46,2 & 39,6 & 34,8 & 40,5 & 61,5 \\
\hline 2005 & 51,1 & 78,1 & 52,2 & 60,0 & 55,0 & 55,1 & 66,5 \\
\hline 2006 & 40,0 & 77,6 & 42,9 & 42,1 & 51,7 & 58,1 & 63,2 \\
\hline 2007 & 56,7 & 77,5 & 47,4 & 45,8 & 36,4 & 70,3 & 65,9 \\
\hline 2008 & 54,5 & 74,6 & 50,0 & 54,8 & 37,1 & 70,8 & 63,9 \\
\hline 2009 & 40,0 & 71,8 & 40,0 & 44,7 & 65,8 & 79,5 & 62,7 \\
\hline 2010 & 41,9 & 76,1 & 31,3 & 42,6 & 41,9 & 73,2 & 62,9 \\
\hline 2011 & 35,0 & 81,1 & 39,1 & 47,9 & 41,4 & 62,1 & 63,6 \\
\hline 2012 & 58,5 & 77,4 & 27,8 & 51,5 & 54,2 & 79,5 & 67,1 \\
\hline 2013 & 48,1 & 80,7 & 11,1 & 59,1 & 53,3 & 82,6 & 69,5 \\
\hline 2014 & 44,4 & 84,0 & 12,5 & 54,8 & 23,5 & 73,9 & 66,4 \\
\hline 2015 & 32,7 & 88,8 & 40,0 & 55,7 & 17,4 & 70,3 & 67,4 \\
\hline 2016 & 49,0 & 81,4 & 40,9 & 58,5 & 20,0 & 72,2 & 65,9 \\
\hline 2017 & 27,8 & 73,8 & 33,3 & 55,1 & 33,3 & 66,7 & 56,8 \\
\hline
\end{tabular}

As taxas de homicídios por arma de fogo em homens jovens no estado revelaram o ano de 2014 (97,4/100.000) com maior taxa, seguido de 2013 (88,4/100.000) e 2000 (82,4/100.000). Os dois primeiros anos de estudo apresentaram as menores taxas, registrando 48,9/100.000 em $1996 \mathrm{e}$ 48,1/100.000 em 1997 (Tabela 2).

Ao considerar as taxas de mortalidade padronizadas entre as Macrorregiões de Saúde do estado, observou-se que a Macrorregião Centro Norte lidera a mortalidade por arma de fogo na maioria dos anos, em que atingiu taxa superior a 110/100.000. Destaca-se o ano de 2014, em que foi registrada a maior taxa do período entre todas as macrorregiões $(158,5 / 100.000)$ enquanto a menor taxa ocorreu em 2013, na macrorregião Leste (Tabela 2). 


\section{Tabela 2}

Taxa padronizada* de homicídios de homens jovens por arma de fogo, segundo macrorregião de saúde. Mato Grosso, 1996-2017.

\begin{tabular}{cccccccc}
$\begin{array}{c}\text { Ano do } \\
\text { óbito }\end{array}$ & Leste & $\begin{array}{c}\text { Centro } \\
\text { Norte }\end{array}$ & Norte & Oeste & Sul & $\begin{array}{c}\text { Centro } \\
\text { Noroeste }\end{array}$ & $\begin{array}{c}\text { Mato } \\
\text { Grosso } \\
\text { (Total) }\end{array}$ \\
\cline { 2 - 7 } $\mathbf{1 9 9 6}$ & 28,3 & 73,4 & 23,0 & 77,1 & 45,7 & 23,4 & $\mathbf{4 8 , 9}$ \\
$\mathbf{1 9 9 7}$ & 25,4 & 69,3 & 31,0 & 90,6 & 33,0 & 21,2 & $\mathbf{4 8 , 1}$ \\
$\mathbf{1 9 9 8}$ & 10,3 & 118,4 & 33,6 & 79,1 & 26,0 & 36,5 & $\mathbf{6 2 , 4}$ \\
$\mathbf{1 9 9 9}$ & 21,7 & 112,6 & 23,3 & 46,0 & 29,4 & 30,5 & $\mathbf{5 5 , 6}$ \\
$\mathbf{2 0 0 0}$ & 59,1 & 155,1 & 34,7 & 56,8 & 51,1 & 46,7 & $\mathbf{8 2 , 4}$ \\
$\mathbf{2 0 0 1}$ & 33,9 & 146,5 & 24,2 & 27,3 & 39,7 & 33,9 & $\mathbf{6 8 , 3}$ \\
$\mathbf{2 0 0 2}$ & 43,0 & 112,9 & 38,1 & 40,7 & 48,6 & 52,8 & $\mathbf{6 6 , 4}$ \\
$\mathbf{2 0 0 3}$ & 18,2 & 110,6 & 39,7 & 39,4 & 33,4 & 50,9 & $\mathbf{6 1 , 1}$ \\
$\mathbf{2 0 0 4}$ & 32,3 & 102,0 & 26,5 & 19,5 & 25,5 & 49,9 & $\mathbf{5 3 , 7}$ \\
$\mathbf{2 0 0 5}$ & 28,7 & 106,5 & 36,9 & 27,2 & 43,9 & 42,1 & $\mathbf{5 9 , 0}$ \\
$\mathbf{2 0 0 6}$ & 34,7 & 121,1 & 34,8 & 36,9 & 28,5 & 32,6 & $\mathbf{6 1 , 3}$ \\
$\mathbf{2 0 0 7}$ & 21,3 & 108,8 & 28,4 & 32,7 & 42,7 & 28,2 & $\mathbf{6 2 , 3}$ \\
$\mathbf{2 0 0 8}$ & 30,6 & 107,9 & 38,8 & 49,4 & 55,3 & 44,4 & $\mathbf{6 0 , 2}$ \\
$\mathbf{2 0 0 9}$ & 31,2 & 115,2 & 41,3 & 70,0 & 56,6 & 37,0 & $\mathbf{6 7 , 8}$ \\
$\mathbf{2 0 1 0}$ & 14,6 & 121,4 & 35,3 & 51,0 & 81,8 & 43,3 & $\mathbf{6 8 , 6}$ \\
$\mathbf{2 0 1 1}$ & 21,8 & 128,0 & 43,3 & 46,1 & 69,7 & 29,5 & $\mathbf{7 0 , 6}$ \\
$\mathbf{2 0 1 2}$ & 11,7 & 127,1 & 62,0 & 36,3 & 92,5 & 65,4 & $\mathbf{8 1 , 4}$ \\
$\mathbf{2 0 1 3}$ & 4,7 & 111,7 & 92,8 & 52,9 & 152,1 & 44,8 & $\mathbf{8 8 , 4}$ \\
$\mathbf{2 0 1 4}$ & 14,3 & 158,5 & 77,2 & 66,4 & 142,2 & 36,7 & $\mathbf{9 7 , 4}$ \\
$\mathbf{2 0 1 5}$ & 16,5 & 140,2 & 79,5 & 41,9 & 89,9 & 27,3 & $\mathbf{8 0 , 8}$ \\
$\mathbf{2 0 1 6}$ & 22,5 & 117,6 & 73,5 & 38,0 & 111,5 & 52,7 & $\mathbf{7 9 , 0}$ \\
$\mathbf{2 0 1 7}$ & 20,4 & 59,6 & 89,3 & 49,9 & 113,1 & 46,2 & $\mathbf{6 5 , 3}$ \\
\hline
\end{tabular}

*por 100.000 homens

Foi observada tendência crescente e significativa da taxa de homicídio por arma de fogo, no período de 1996 a 2017, para as macrorregiões Norte, Oeste, Sul e para o estado de Mato Grosso. As macrorregiões Leste, Centro Norte e Centro Noroeste as taxas mantiveram estáveis ao mesmo tempo. Não se observou tendência decrescente em nenhuma macrorregião (Tabela 3). 


\section{Tabela 3}

Tendência temporal dos homicídios por arma de fogo em homens jovens, segundo macrorregião de saúde. Mato Grosso, 1996 a 2017.

\begin{tabular}{|c|c|c|c|c|c|}
\hline Macrorregião & Parâmetro & $\begin{array}{l}\text { Coeficiente } \\
\text { (IC 95\%) }\end{array}$ & Tendência & $p$ & $p^{1}$ \\
\hline Leste & $\begin{array}{l}\text { Termo AR1 } \\
\text { Constante }\end{array}$ & $\begin{array}{c}0,28(-0,35-13,98) \\
24,71(16,81 ; 32,72)\end{array}$ & Estável & $\begin{array}{l}0,379 \\
<0,001\end{array}$ & 0,990 \\
\hline Centro Norte & $\begin{array}{l}\text { Termo AR1 } \\
\text { Constante }\end{array}$ & $\begin{array}{c}0,49(-0,05 ; 1,02) \\
110,89(94,25 ; 127,52)\end{array}$ & Estável & $\begin{array}{c}0,073 \\
<0,001\end{array}$ & 0,922 \\
\hline Norte & $\begin{array}{l}\text { Termo AR1 } \\
\text { Constante }\end{array}$ & $\begin{array}{c}0,89(0,56 ; 1,21) \\
50,16(13,07 ; 87,25)\end{array}$ & Crescente & $\begin{array}{c}<0,001 \\
0,008\end{array}$ & 0,855 \\
\hline Oeste & $\begin{array}{l}\text { Termo AR1 } \\
\text { Constante }\end{array}$ & $\begin{array}{c}0,59(0,17 ; 1,00) \\
50,56(36,01 ; 65,09)\end{array}$ & Crescente & $\begin{array}{l}0,006 \\
<0,001\end{array}$ & 0,915 \\
\hline Sul & $\begin{array}{l}\text { Termo AR1 } \\
\text { Constante }\end{array}$ & $\begin{array}{c}0,82(0,55 ; 1,09) \\
68,65(8,75 ; 128,56)\end{array}$ & Crescente & $\begin{array}{c}<0,001 \\
0,025\end{array}$ & 0,907 \\
\hline \multirow[t]{2}{*}{$\begin{array}{l}\text { Centro } \\
\text { Noroeste }\end{array}$} & Termo AR1 & $0,11(-0,48 ; 0,69)$ & Estável & 0,718 & 0,482 \\
\hline & Constante & $39,76(33,54 ; 45,98)$ & & $<0,001$ & \\
\hline $\begin{array}{c}\text { Mato Grosso } \\
\text { (Total) }\end{array}$ & $\begin{array}{l}\text { Termo AR1 } \\
\text { Constante } \\
\end{array}$ & $\begin{array}{c}0,68(0,41 ; 0,96) \\
65,95(54,11 ; 77,77)\end{array}$ & Crescente & $\begin{array}{l}<0,001 \\
<0,001\end{array}$ & 0,801 \\
\hline
\end{tabular}

${ }^{1}$ Teste de Box e Pierce

A validação do modelo ajustado da taxa padronizada de homicídios por arma de fogo de homens jovens segundo Macrorregiões de Saúde do estado de Mato Grosso, Brasil, se deu a partir da comparação dos valores reais das taxas padronizadas calculadas de 1996 a 2017, com os valores previstos pelo modelo AR (1) para esse mesmo período. Dessa forma, observa-se que as previsões foram bastante razoáveis, de forma que o modelo estimado acompanhou o padrão observado das 
taxas reais. A figura 1 apresenta o gráfico com a série original e predita com base no modelo ajustado, demonstrando os valores estimados próximos aos reais, confirmando o bom ajuste do modelo.

\section{Figura 1}

Série original e série predita com base no modelo auto regressivo de ordem 1 da taxa padronizada de homicídios por arma de fogo de homens jovens, segundo Macrorregiões de Saúde. Mato Grosso, Brasil, 1996-2022.

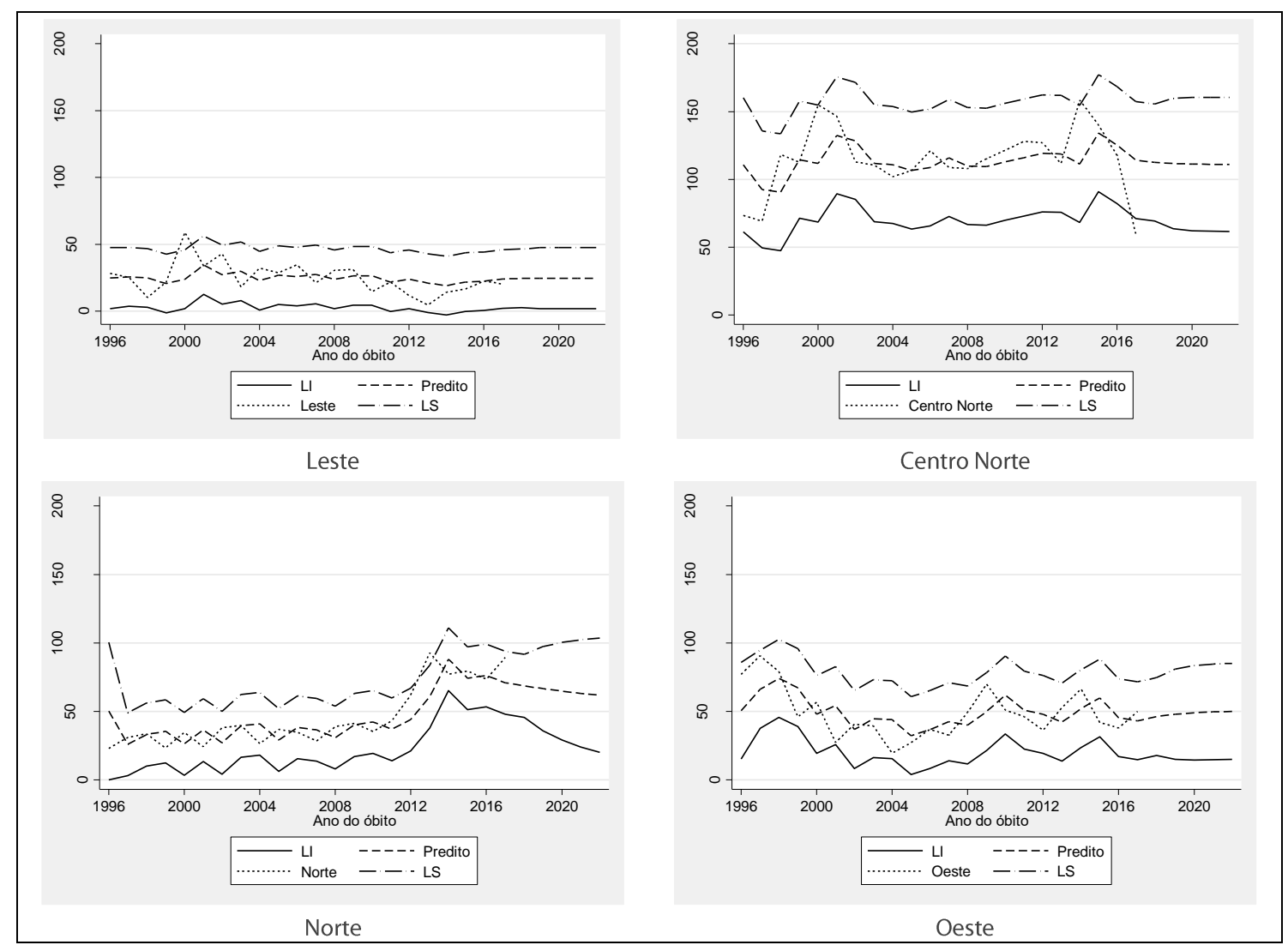




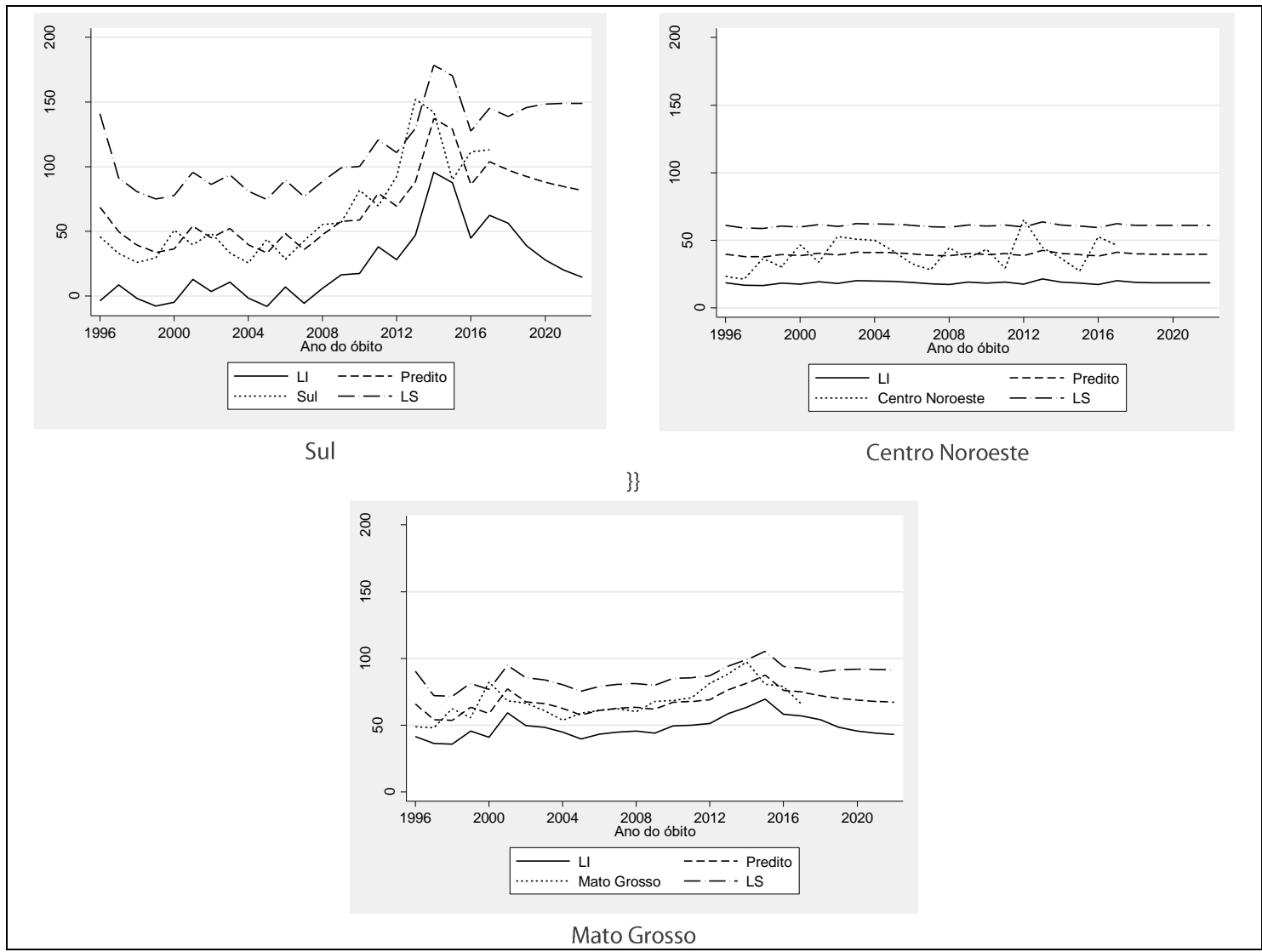

Limite inferior; LS: Limite Superior

\section{Discussão}

Os dados encontrados neste estudo desvelam o cenário dos homicídios em homens jovens por arma de fogo nas macrorregiões de saúde do estado do Mato Grosso. Trata-se de um estado da Amazônia Legal com vasta extensão territorial e diferenças regionais marcantes, que consequentemente refletem o comportamento das taxas de homicídio, sobretudo na população estudada.

Foram encontradas elevadas taxas de homicídio nas macrorregiões de saúde, com tendência crescente nas macrorregiões Norte, Oeste e Sul, evidenciando contrastes entre as áreas estudadas. Mesmo que algumas macrorregiões apresentassem taxas inferiores, destaca-se a tendência crescente para metade (seis) delas e para o estado. Ressalta-se ainda o fato de que para as demais, a 
tendência se mostrou estável, ou seja, no período de 1996 a 2017, nenhuma macrorregião apresentou tendência decrescente da taxa de mortalidade por homicídios por arma de fogo em homens jovens.

Cerqueira et al. (2020) explicam que o crescimento dos homicídios no Brasil ao longo dos anos ocorreu basicamente devido às mortes com o uso das armas de fogo, ao passo que as mortes por outros meios permaneceram constantes desde o início dos anos 1990. Entre 1980 e 2016, cerca de 910 mil pessoas foram mortas com o uso de armas de fogo. No início dos anos 1980, 40,0\% das pessoas assassinadas no Brasil eram vítimas de armas de fogo e, em 2017, essa proporção cresceu para 72,4\% (Ministério da Saúde, 2019).

Os homicídios envolvendo homens jovens tendem a ser mais afetados por desenvolvimentos sociopolíticos, mercados de drogas e outros fatores voláteis que causam picos nas mortes (UNODC, 2019). Nesta perspectiva buscou-se compreender o que ocorre nas macrorregiões de saúde de Mato Grosso, observando que aquelas que apresentaram tendência crescente detêm características semelhantes e particulares que, apesar de representarem fortalecimento econômico, trazem consigo alguns ônus, nesse caso, a mortalidade da juventude masculina por armas de fogo.

A macrorregião Norte é composta por regiões em processo de urbanização intenso, de avanço da fronteira agrícola e exploração de garimpo. A macrorregião Sul também sofre consequências da urbanização, abrigando um polo industrial importante para o estado. Tais características podem estar relacionadas às taxas de homicídios por armas de fogo (Koga et al., 2008; Melo et al., 2017; Silva \& Bampi, 2019), como as reveladas neste estudo.

A macrorregião Oeste abarca parte da fronteira do país com a Bolívia, além de enfrentar questões importantes com o garimpo ilegal (Scatena et al., 2014; Martinelli, 2014). Nesse sentido, achados semelhantes do estudo de Nogueira et al. (2020) corroboram esses resultados quando descrevem que houve predomínio do uso de armas de fogo e taxas de homicídios mais elevadas em regiões fronteiriças do estado do Paraná, especialmente entre jovens do sexo masculino. 
Ademais da tendência crescente das taxas de homicídios por armas de fogo no período de 1996 a 2017, a macrorregião Oeste também se destaca apresentando o maior crescimento até 2022. Os jovens do sexo masculino são predominantemente afetados por homicídios quando altos níveis de violência são provocados pelo crime organizado (UNODC, 2019). O conflito entre os envolvidos é resolvido com truculência, pagando-se com a própria vida e na maioria das vezes, utilizando arma de fogo, que funciona como símbolo de poder (Carneiro, 2009; Cepik \& Borba, 2011; Metzl, 2019). Esse cenário está reproduzido na macorrergião Oeste, que reluta contra o crime organizado presente na região que opera o tráfico internacional de drogas e armas na fronteira com a Bolívia.

Além das questões particulares de cada macrorregião, a difusão das armas de fogo tratada globalmente deve ser considerada, já que há consenso na literatura científica internacional sobre os efeitos danosos da propagação de armas de fogo na sociedade (Bauchner et al., 2017; Donohue et al., 2019).

Em estudo de revisão sistemática, Santaella-Tenorio et al. (2016) analisaram trabalhos que exploram a associação entre leis relacionadas ao uso de armas de fogo e homicídios e concluíram que dos 130 estudos de dez países, a implementação simultânea de leis direcionadas a restrição de armas de fogo foi associada à redução dos homicídios. Outrossim, estudos nacionais referem que o relaxamento da legislação brasileira em vigor sobre o controle do acesso às armas de fogo implica em mais mortes e ainda mais insegurança no país (Cerqueira \& Mello, 2013; Waiselfisz, 2016).

O Estatuto do Desarmamento brasileiro, instituído pela Lei n. 10.826 (2003), estabeleceu critérios mais rigorosos para o controle de armas de fogo em circulação na tentativa de reduzir as taxas de homicídios. Ministério Filho et al. (2017) enfatizam que em dois anos de promulgação, as campanhas nacionais foram responsáveis por retirar de circulação cerca de 570 mil armas de fogo e as taxas de homicídios caíram, inclusive no estado de Mato Grosso, pois além de disciplinar a posse e o porte de armas de fogo no território nacional e estabelecer penas para condutas ilícitas relacionadas à matéria, a lei previu campanhas de desarmamento. 
Nesse sentido, a posse civil de armas de fogo está positivamente correlacionada com a taxa de homicídio, embora possa se tornar secundária a outros fatores de natureza socioeconômica, principalmente a desigualdade de renda. A regulamentação mais rigorosa da posse de armas de fogo, juntamente com os esforços para reduzir o número, pode ajudar a reduzir a taxa de homicídios (UNODC, 2019). No entanto, autores referem que a redução notável da taxa de homicídio só pode ser obtida se outros fatores forem abordados ao mesmo tempo (Malta et al., 2018),

O estudo de Freire (2018) em que relaciona a redução das taxas de homicídios de São Paulo e as Políticas Públicas implantadas sugere que, embora não seja possível estimar o efeito do tratamento de cada política específica implementada entre os anos 1990 e 2010, o impacto agregado certamente não seja desprezível, uma vez que as taxas de homcídios caíram 70\% nesse período. Desta forma, o estado de São Paulo oferece um exemplo de que é possível combater o crime com políticas direcionadas.

Lima et al. (2015) questionam as tentativas de conter a violência no Brasil afirmando que avanços eventuais na gestão policial e reformas na legislação penal têm se revelado insuficientes para reduzir a incidência da violência urbana, com evidência de falta de coordenação e controle. No âmbito do Congresso Nacional, há dificuldades para fazer avançar uma agenda de reformas requerida pela Constituição de 1988, que até hoje possui diversos artigos sem regulamentação, o que permite tanto zonas de sombra e insegurança jurídica como a prevalência de práticas institucionais forjadas nos períodos autoritários, com o discurso de ódio, lobby de armas, além da naturalização da violência (Gamlin \& Hawkes, 2018).

Além disso, Barros et al. (2017) apresentam que articuladamente à tradição escravocrata e elitista, tem-se aí uma possível chave analítica frente à aparente naturalização com que parcela significativa da sociedade brasileira admite, e até demanda, o extermínio de certas juventudes no Brasil associadas à pobreza, ao risco e à criminalidade, em pleno "regime democrático".

A violência se torna objeto do setor saúde pelo impacto que provoca na qualidade de vida individual e coletiva, pelas lesões físicas, psíquicas e morais que acarreta e pelas exigências de atenção e 
cuidados dos serviços médico-hospitalares (Minayo et al., 2018). A perda por homicídio gera consequências significativas de curto e longo prazo, com custos emocionais e sociais, tanto para a sociedade quanto para os familiares e amigos das vítimas, além dos anos de vida perdidos (Ministério da Saúde, 2005; Costa et al., 2017), refletindo assim forte impacto na saúde das pessoas.

Embora haja contexto de relativos avanços sobre a violência no campo da saúde, Martins e Fernandes (2016) reforçam que as mortes violentas não terem diminuído demonstram insuficiência dos avanços das políticas para reverter a realidade.

É válido reconhecer que este estudo foi interpretado à luz das limitações comuns decorrentes do uso de dados secundários, seja refente à subnotificação ou a qualidade do registro da causa básica do óbito, representada principalmente pelo volume de registros de causas indeterminadas (Ministério da Saúde, 2019). Contudo, sob este aspecto, ressalta-se que no período de 2007 a 2017 houve redução dessas causas no estado de Mato Grosso (Minstério da Saúde, 2019). Outrossim, o número de estudos primários sobre as regiões de saúde de Mato Grosso limitaram a discussão de determinados aspectos e características dos locais estudados com os resultados aportados neste estudo, constatando a necessidade precípua de aprofundamento no tema.

\section{Conclusão}

A tendência crescente das taxas de homicídios por armas de fogo em homens jovens nas macrorregiões Norte, Oeste e Sul, tal como no estado de Mato Grosso, evidencia a necessidade de atuação mais específica, interdisciplinar, multiprofissional e intersetorial visando contribuir efetivamente para discussão e prevenção dos homicídios por armas de fogo nesta população. Isto posto, a contribuição desta pesquisa visa dar visibilidade aos homicídios por arma de fogo em homens jovens e às áreas identificadas com tendência de crescimento das taxas, oferecendo subsídios importantes para o planejamento de políticas públicas voltadas à saúde e à segurança pública. 
Enfrentar os homicídios por armas de fogo de homens jovens significa reconhecer as complexas forças estruturais, históricas, políticas, sociais e econômicas que impulsionam o continuum da violência em todas as suas formas. Destarte, os resultados deste estudo indicam que se as estratégias não forem mudadas, as taxas de homicídios de homens jovens por armas de fogo em Mato Grosso continuarão altas ou crescentes.

Por fim, espera-se ter suscitado a discussão sobre uma temática pertinente e que os resultados apoiem a elaboração de outros estudos sobre tendência de homicídios de homens jovens, pois estudos dessa natureza contribuem para a tomada de decisão e para o fortalecimento das ações de controle. Fia-se que sirva de recurso para a criação de políticas públicas capazes de envolver segurança pública, a saúde e sociedade civil, com o propósito de trabalhar conjuntamente em todas as dimensões que o enfrentamento dos homicídios requer.

\section{Referências}

Barros, J. P. P., Benício, L. F. S., Silva, D. B., Leonardo, C. S., \& Torres, F. J. (2017) Homicídios juvenis e os desafios à democracia brasileira: implicações ético-políticas da psicologia. Psicologia: Ciência e Profissão, 37(04), 1051-1065. https://doi.org/10.1590/1982-3703002892017

Bauchner, H., Rivara, F. P., Bonow, R. O., Bressler, N. M., Disis, M. L., Heckers, S., Josephson, A., Kibbe, M. R., ... \& Robinson, J. K. (2017) Death by Gun Violence: A Public Health Crisis. JAMA, 318(18), 1763-176. doi:10.1001/jama.2017.16446

Box, G. E. P., Jenkins, G. M., Reinsel, G. C. \& Ljung, G. M (2015). Time series analysis forecasting and control (5 ed.). San Francisco, Estados Unidos: Wiley.

Carneiro, L. P. (2009). Violência Urbana, Segurança Pessoal e Criminalidade. In: Cardoso FH \& Foxley A (Org.), América Latina: desafios da democracia e do desenvolvimento, políticas sociais para além da pobreza. (v. 2. pp. 242-270). Elsevier.

Cepik, M., \& Borba, P. (2011). Crime organizado, estado e segurança internacional. Contexto Internacional, 33(02), 375-405. DOI: http://dx.doi.org/10.1590/S0102-85292011000200005

Cerqueira, D., \& Mello, J. M. P. (2013). Evaluating a national anti-firearm law and estimating the causal effect of guns on crime [Texto para Discussão n. 607]. Rio de Janeiro, Brasil: PUC. 
Cerqueira, D., Bueno, S., Alves, P.P., Lima, R. S., Silva, E. R. A., Ferreira, H., Pimentel, A., ... e Figueiredo, T. (2020). Atlas da Violência 2020. Rio de Janeiro, Brasil: Ipea. Recuperado de https://www.ipea.gov.br/portal/images/stories/PDFs/relatorio_institucional/200826_ri_atl as_da_violencia.pdf

Costa, D. H., Schenker, M., Njaine, K., \& Souza, E. R. (2017). Homicídios de jovens: os impactos da perda em famílias de vítimas. Physis: Revista de Saúde Coletiva, 27(03), 685-705. DOI: http://dx.doi.org/10.1590/s0103-73312017000300016.

Doll, R., Payne P., \& Waterhouse, J. A. H. (1966). Cancer Incidence in Five Continents, (vol. I). Berlin, Alemenia: Union Internationale Contre le Cancer.

Donohue, J.J.; Aneja, A.; \& Weber, K. D. (2019). Right-to-carry laws and violent crime: a comprehensive assessment using panel data and a state-level synthetic control analysis. Journal of Empirical Legal Studies, 16(02), 198-247. Recuperado de https://www.nber.org/papers/w23510.pdf

Freire, D. (2018). Evaluating the Effect of Homicide Prevention Strategies in São Paulo, Brazil: A Synthetic Control Approach. Latin American Research Review, 53(2), 231-249. DOI: http://doi.org/10.25222/larr.334

Gamlin, J. B.; \& Hawkes, S. J. (2018). Masculinities on the Continuum of Structural Violence: The Case of Mexico's Homicide Epidemic. Social Politics: International Studies in Gender, State \& Society, 25(01), 50-71. DOI: https://doi.org/10.1093/sp/jxx010

Geneva Declaration on Armed Violence and Development. (2019). Recuperado de http://www.genevadeclaration.org/the-geneva-declaration/what-is-the-declaration.html

Instituto Brasileiro de Geografia e Estatística. (2020). População. Recuperado de https://www.ibge.gov.br/cidades-e-estados/mt.html

Kalesan B, Mobily ME, Keiser O, Fagan JA, \& Galea S. (2016) Firearm legislation and firearm mortality in the USA: a cross-sectional, state-level study. Lancet, 387(10030), 1847-1855. DOI: https://doi.org/10.1016/S0140-6736(15)01026-0

Koga, D., Ramos, F., \& Nakano, K. (2008). A disputa territorial redesenhando relações sociais nas cidades brasileiras. Serviço e Sociedade, 94(01), 01-19.

Krug, E.G., Dahlberg, L. L., Mercy, J. A., Zwi, A. B., \& Lozano, R. (2002). World report on violence and health. Geneva: World Health Organization. Recuperado de http://www.who.int/violence_injury_prevention/violence/world_report/en/

Lee, L. K., Fleegler, E. W., Farrell C., Avakame' E., Srinivasan, S., Hemenway, D., \& Monuteaux, M. C. (2017). Firearm Laws and Firearm Homicides: A Systematic Review. JAMA Intern Med., 177(1), 106-119. doi:10.1001/jamainternmed.2016.7051 
Lei no 10.826, de 22 de dezembro de 2003. Dispõe sobre registro, posse e comercialização de armas de fogo e munição, sobre o Sistema Nacional de Armas - Sinarm, define crimes e dá outras providências. Recuperado de www.planalto.gov.br/ccivil_03/leis/2003/I10.826.htm

Lima, R. S.; Sinhoretto, J.; \& Bueno, S. (2015). A gestão da vida e da segurança pública no Brasil. Soc. estado, 30(1), 123-144. http://dx.doi.org/10.1590/S0102-69922015000100008

Malta, D. C., Minayo, M. C. S., Soares Filho, A. M., Silval, M. M. A., Montenegro, M. M. S., Ladeira, R. M., Morais Neto, O. L., ... \& Naghavi, M. (2017). Mortalidade e anos de vida perdidos por violências interpessoais e autoprovocadas no Brasil e Estados: análise das estimativas do Estudo Carga Global de Doença, 1990 e 2015. Revista Brasileira de Epidemiologia, 20(supl. 1), 142-156. https://dx.doi.org/10.1590/1980-5497201700050012

Martinelli, N. L. A. (2014). Regionalização da saúde no Estado de Mato Grosso: o processo de implementação e a relação público-privada na região de saúde do Médio Norte Matogrossense (Tese de doutorado). Faculdade de Medicina da Universidade de São Paulo, São Paulo, Brasil.

Martins, A. C., \& Fernandes, C. R. (2016). Mortalidade por agressões e lesões autoprovocadas voluntariamente: reflexões sobre a realidade brasileira. Saúde em foco, 01(01), 01-12. Recuperado de https://smsrio.org/revista/index.php/revsf/article/view/163

Melo, A. C. M., Silva, G. D. M., \& Garcia, L. P. (2017). Mortalidade de homens jovens por agressões no Brasil, 2010-2014: estudo ecológico. Cadernos de Saúde Pública, 33(11), 1-15. DOI: http://dx.doi.org/10.1590/0102-311x00168316

Mello Jorge, M. H. P., Laurenti R, Gotlieb, S. L. D. (2009). O sistema de informações sobre mortalidade - SIM: concepção, implantação e avaliação. In A experiência brasileira em sistemas de informação em saúde. Brasília, Brasil: Ministério da Saúde.

Metzl, J. What guns mean the symbolic lives of firearms? (2019). Palgrave Communications, 05(35), 1-5. DOI: https://doi.org/10.1057/s41599-019-0240-y

Minayo, M. C. S. (2013). Violência e Educação: impactos e tendências. Revista Pedagógica, 15(31), 249264. DOI: https://doi.org/10.22196/rp.v15i31.2338

Minayo, M. C. S., Souza, E. R., Silva, M. M. A., \& Assis, S. G. (2018). Institucionalização do tema da violência no SUS: avanços e desafios. Ciência e Saúde Coletiva, 23(06), 2007-2016. DOI: http://dx.doi.org/10.1590/1413-81232018236.04962018

Ministério da Saúde (2005). Secretaria de Vigilância em Saúde. Impacto da violência na saúde dos brasileiros. Brasília, Brasil: Ministério da Saúde. 
Ministério da Saúde (2019). Departamento de Informática do Sistema Único de Saúde. Informações de Saúde. Brasília, Brasil: Sistema de Informações sobre Mortalidade Recuperado de http://tabnet.datasus.gov.br.

Ministério da Saúde (2011). Secretaria de Vigilância em Saúde. Departamento de Análise de Situação de Saúde. Manual de Instruções para o preenchimento da Declaração de óbitos. Brasília, Brasil: Ministério da Saúde.

Ministério dos Direitos Humanos (2018). Secretaria Nacional de Proteção dos Direitos da Criança e Adolescente. Letalidade infanto-juvenil: dados da violência e políticas públicas existentes. Brasília, Brasil: DF.

Ministério Filho, M. A. P., Alves, U. J., \& Tameirão, J. (2017). O papel do estado na garantia de segurança e o estatuto do desarmamento: uma breve análise das cidades mineiras de Teófilo Otoni, Novo Cruzeiro e Padre Paraíso. Revista Científica Fenord, 07(01), 212-243. Recuperado de http://www.fenord.edu.br/revistaaguia/revista2017/textos/artigo08.pdf

Nogueira, V. Denepotti, Xavier-Gomes, L. M. \& Barbosa, T. L. A. (2020). Mortalidade por homicídios em linha de fronteira no Paraná, Brasil. Ciência \& Saúde Coletiva, 25(8), 3107-3118. https://doi.org/10.1590/1413-81232020258.28522018.

Santaella-Tenorio, J., Cerdá, M., Villaveces, A., \& Galea, S. (2016). What Do We Know About the Association Between Firearm Legislation and Firearm-Related Injuries? Epidemiologic Reviews, 38(01), 140-157. https://doi.org/10.1093/epirev/mxv012

Scatena, J. H. G., Oliveira, L. R., Galvão, N. D., \& Neves, M. A. B. (2014). Caracterização das regiões de saúde de Mato Grosso. In: Scatena JHG, Kehrig RT, Spinelli MAS Eds.), Regiões de Saúde: Diversidade e processo de regionalização em Mato Grosso. (pp. 135-168). São Paulo, Brasil: Hucitec.

Secretaria de Estado de Saúde. (2018). Panorama das Regiões de Saúde II -MT. Cuiabá, Brasil: Secretaria Adjunta de Atenção à Saúde.

Segi, M. (1960). Cancer Mortality for Selected Sites in 24 Countries (1950-57). Sendai: Japon. Department of Public Health, Tohoku University of Medicine.

Silva, C. A. F., \& Bampi, A. C. S. (2019). Regional Dynamics of the Brazilian Amazon between modernization and land conflicts. Cuadernos de Geografia: Revista Colombiana de Geografia, 28(02), 340-356. https://doi.org/10.15446/rcdg.v28n2.72872

Simonetti J. A., Rowhani-Rahbar A., Mills B, Young B., \& Rivara F.P. (2015). State Firearm Legislation and Nonfatal Firearm Injuries. Am J Public Health, 105(8), 1703-1709. https://doi.org/10.2105/AJPH.2015.302617 
United Nations Office on Drugs and Crime. (2019). Global study on homicide. Vienna, Austria. Recuperado de https://www.unodc.org/documents/data-and-analysis/gsh/Booklet1.pdf

Waiselfisz, J. J. (2016). Mapa da Violência 2016: Homicídios por armas de fogo no Brasil. São Paulo, Brasil: FLACSO.

World Health Organization. (2018). World health statistics 2018: monitoring health for the SDGs, Sustainable Development Goals. Recuperado de https://www.who.int/gho/publications/world_health_statistics/en/

Zeoli, A. M., Goldstick, J., Mauri, A., Wallin, M., Goyal, M. \& Cunningham, R. (2019). The association of firearm laws with firearm outcomes among children and adolescents: a scoping review. Journal of Behavioral Medicine, 42(1), 741-762. https://doi.org/10.1007/s10865-019-00063y 


\section{Población y Salud en Mesoamérica}

¿Quiere publicar en la revista?

Ingrese aquí

O escríbanos:

revista@ccp.ucr.ac.cr

\section{$1^{\circ}$ Revista}

Electrónica UCR

13 años de liderazgo

$\square$

Enlace Award, 2007

\section{UCR Index}

Población y Salud en Mesoamérica (PSM) es la revista electrónica que cambió el paradigma en el área de las publicaciones científicas electrónicas de la UCR. Logros tales como haber sido la primera en obtener sello editorial como revista electrónica la posicionan como una de las más visionarias.

\section{Revista PSM es la letra delta mayúscula, el cambio y el futuro.}

Indexada en los catálogos más prestigiosos. Para conocer la lista completa de índices, ingrese aquí.

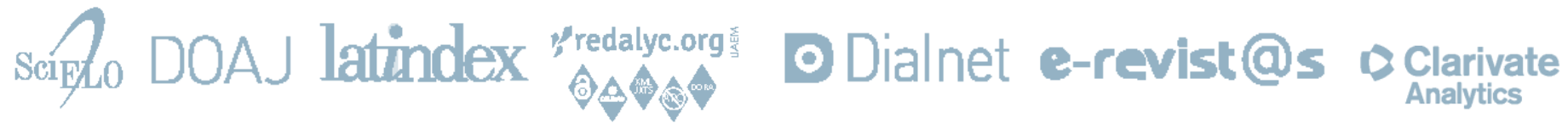

f Revista Población y Salud en Mesoamérica - PSM

Centro Centroamericano de Población

Universidad de Costa Rica

\section{(웅(-)}

